\title{
New Electrorheological Fluid: Theory and Experiment
}

\author{
Wing Yim Tam, Guang Hua Yi, Weijia Wen, Hongru Ma,* M. M. T. Loy, and Ping Sheng \\ Department of Physics, The Hong Kong University of Science and Technology, Clear Water Bay, Kowloon, Hong Kong
}

(Received 10 October 1996)

\begin{abstract}
We have fabricated novel doubly coated particles, with a conducting inner layer and an insulating outer layer, for use in electrorheological fluids. Orders of magnitude enhancement in the yield stress is obtained by using such particles, when compared to that of bare particles or singly coated particles (with the insulating layer only). The measured static yield stress agrees well with first-principles calculations, and supports a physical picture using electrostatic energy arguments for an intuitive understanding of this large enhancement. [S0031-9007(97)02932-3]
\end{abstract}

PACS numbers: $61.90 .+\mathrm{d}, 41.20 . \mathrm{Cv}, 62.20 .-\mathrm{x}$

Electrorheological (ER) fluids, which can change from a liquidlike state to a solidlike viscous state upon the application of an electric field [1], hold great potential for many applications such as in shock-absorbing vibrational dampers, clutches, and robotics [2]. In practice, however, large scale utilization of ER fluids has yet to be realized for lack of ER systems with the required yield stress, stability of the ER effect over an extended period, ER dynamic range, and acceptable wear resistance, among other conditions. Part of the obstacles in developing high performance ER fluids lies in a lack of clear understanding for the ER mechanism and its limiting potential, as well as in the difficulty of finding a single material that can fulfill all the electrical and mechanical requirements of such an ER system. In this Letter, we propose a novel ER system consisting of multiply coated particles in a dielectric fluid. Through both physical arguments and first-principles calculations, we demonstrate that replacing dielectric particles by such particles with inner metallic coatings and outer dielectric coatings will have orders of magnitude enhancement over the otherwise identical ER systems [3]. We have fabricated such particles and we present experimental results demonstrating the expected improvement in yield stress. More importantly, by coupling a physical understanding of the ER mechanism with the experimental fabrication and characterization of the particles, we show that there can be a path of continuous improvement through which optimal ER fluid performance may be achieved.

An intuitive understanding of the ER effect can be obtained by capacitive electrostatic energy argument. Consider two conducting spheres of radius $R$ in close proximity to each other, with a separation $\delta$ between their surfaces at the point of closest approach. Provided $R \gg \delta$, the mutual capacitance has the form

$$
C \propto R \ln \frac{R}{\delta} .
$$

Under an applied electric field $E$ along the axis joining the centers of the two spheres, the leading order term of the free energy density is proportional to $C$. If now we apply a shear perpendicular to the electric field, the line segment joining the centers of the two spheres will be both tilted with an angle $\theta$ with respect to the electric field direction, as well as lengthened. If the new separation is denoted $d$, then $(2 R+d) \cos \theta=2 R+\delta$, so that $d \approx \delta+R \theta^{2}$ for $\theta$ small. Within the small angle approximation, the shear stress is given by $-(\partial C / \partial \theta) / R \propto$ $(R / \delta) \sqrt{(d-\delta) / R}$, which is noted to have a peak value. The static yield stress is defined by the peak value of the shear stress, given by the condition of $d=2 \delta$, so that the static yield stress $\sim \sqrt{R / \delta}\left(\epsilon_{l} E^{2} / 8 \pi\right)$, where the quantity in parentheses is the unit of energy density in the present problem, with $\epsilon_{l}$ denoting the liquid dielectric constant. This heuristic argument tells us that in order to obtain a large ER effect in terms of the yield stress, we should strive to optimize the insulating layer coating thickness so that it would prevent shorting between the conducting spheres and provide wear resistance on the one hand, and give a large yield stress on the other. Since the effect depends on the ratio between $R$ and $\delta$, it is also predicted that the particle size will have an effect. In this regard, a large dielectric constant for the coating material (or the liquid) is a plus, as it increases the capacitance of the system [4].

We now describe the fabrication process for the dielectric/metal (DM) doubly coated particles (DMP). The core particles are solid glass spheres commercially available in various sizes. In this work, we used spheres with diameters $1.5 \mu \mathrm{m}( \pm 0.1 \mu \mathrm{m})$ and $50 \mu \mathrm{m}( \pm 7.5 \mu \mathrm{m})$. From theoretical considerations above, it is clear that the material for the core is immaterial to the ER effect; these spheres have the advantages of high strength and low mass (which is important both for better response time and for avoiding sedimentation from gravity). The conventional electroless plating process [5] was used to deposit the inner conducting nickel layer. The outer insulating layer material used was titanium oxide, chosen for its hardness, resistance to wear, the high dielectric constant. This outer layer was deposited using a sol-gel process [6]. Thickness control of the layers was achieved by solution concentrations and process durations. It is crucial to avoid particle coagulation during the process, and this was achieved by adding an excess sugar solution to form a buffer, which could 
subsequently be removed by heating. The sugar solution, in addition, formed a foam structure during heating which further separated the particles. After heating $\left(500{ }^{\circ} \mathrm{C}\right.$ for 10 hours) to remove all organic components, the DMP's were collected as ashes and dispersed in silicon oil to form the DMP ER fluid. The heating also serves to properly anneal the $\mathrm{TiO}_{2}$ coating, with the very desirable properties of high dielectric constant, excellent adhesion, and hardness.

We have taken cross sectional electron microscope pictures at various stages of the coating process for monitoring purposes. A $1.5 \mu \mathrm{m}$ particle with only the nickel coating is shown in Fig. 1(a). It is seen that the metallic coating was on the order of $100 \AA$ in thickness with excellent uniformity. Figure 1(b) shows a doubly coated particle at the completion of the process (after heating.) In contrast to Fig. 1(a), the $\mathrm{TiO}_{2}$ coating has a much greater variation in thickness, ranging from 100 to $250 \AA$ in this case. Figure 1(c) shows, at higher magnifications, a $50 \mu \mathrm{m}$ doubly coated particle showing the annealed crystalline structure of the $\mathrm{TiO}_{2}$ outer coating. Comparison between Figs. 1(a) and 1(b) or 1(c) also indicated the disappearance of the metal/glass boundary clearly visible in Fig. 1(a). This is most likely due to diffusion of the nickel atoms during the heating process after the $\mathrm{TiO}_{2}$ coating process. However, as we will see in yield stress measurements below, this conducting layer, while not visible in the picture, is sufficiently continuous to fulfill its mission of expelling the electric field (from the interior of the particles) and led to behavior markedly different from particles without such an inner conducting coating. As a further check, we have performed a compositional analysis of the particles. The results are in general agreement with the above picture.

A parallel plate viscometer was used to measure the yield stress of the DMP/silicon oil ER system. To remove any traces of water from the system, all samples went through a 24 hours heating process at $120{ }^{\circ} \mathrm{C}$ prior to each measurement. The yield stress was measured with a $50 \mathrm{~Hz}$ ac field applied between the parallel plates, and using samples with particle/fluid fraction fixed to 0.2. Figures 2(a) and 2(b) show the measured static yield stress for a DMP/silicon oil ER system using the 1.5 and $50 \mu \mathrm{m}$ diameter particles, respectively, as a function of electric field strength. Static yield stress over $2000 \mathrm{~Pa}$ was obtained at $2 \mathrm{kV} / \mathrm{mm}$ for the DMP system. For comparison, we also showed the static yield stress measured for the bare glass particles as well as glass particles coated only with $\mathrm{TiO}_{2}$ [see also Fig. 2(c) with an expanded scale for Fig. 2(a)]. The ER enhancement of the DMP system was over 2 orders of magnitude. It is seen that the DMP system followed generally the expected electric field squared dependence. These DMP's were found to be extremely robust and can sustain both the high electric field and high stress which are prerequisites for any application. The size dependence is seen to be in general agreement with the intuitive picture presented above.

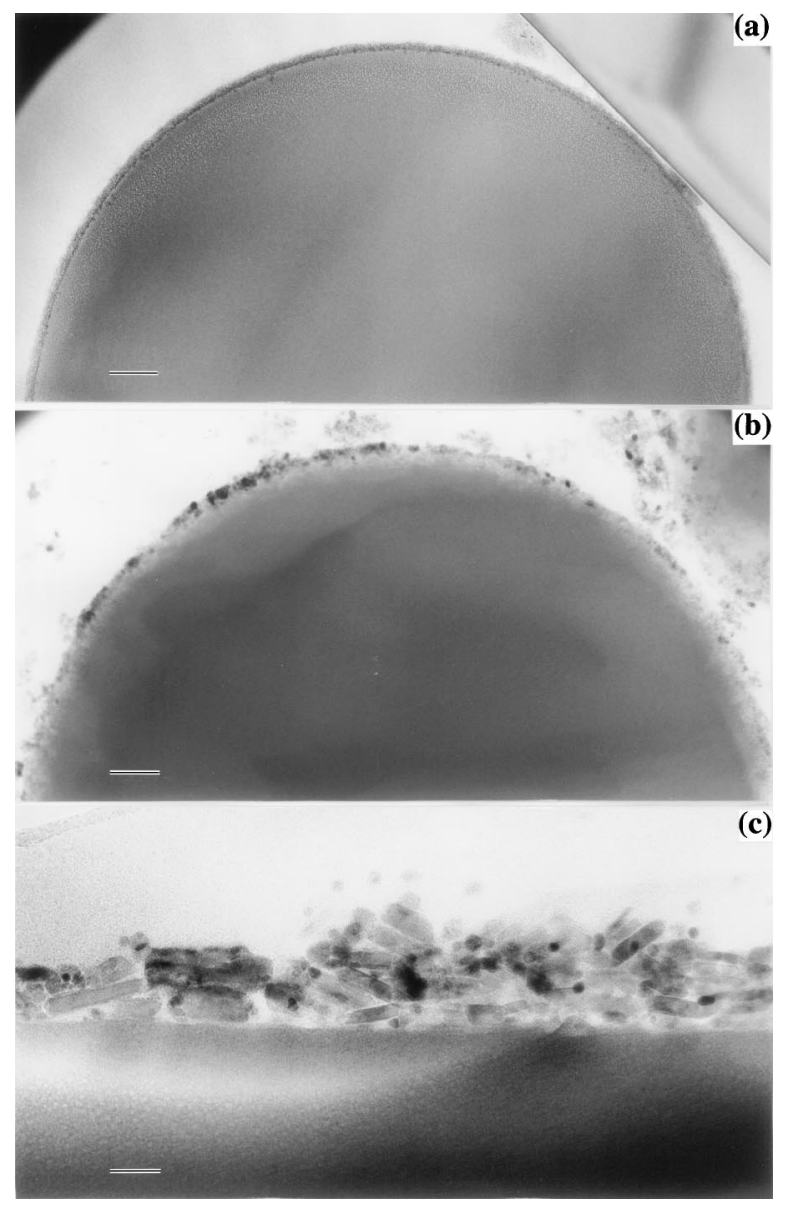

FIG. 1. Cross sectional electron micrographs for (a) $1.5 \mu \mathrm{m}$ nickel coated, (b) $1.5 \mu \mathrm{m}$ doubly coated, and (c) $50 \mu \mathrm{m}$ doubly coated particles. The scale bar is 100 and $25 \mathrm{~nm}$ for (a), (b), and (c), respectively. While in (a), the metal/glass interface is clearly visible, diffusion of $\mathrm{Ni}$ atoms, probably from the heating process following the sol-gel process, blurred this boundary, as shown in (b) and (c). The $\mathrm{TiO}_{2}$ coating thickness is seen to be in the range of 10 to $30 \mathrm{~nm}$ for (b) and 25 to $60 \mathrm{~nm}$ for (c). Note also the darker spots seen only in the $\mathrm{TiO}_{2}$ coatings in (b) and (c). This led us to believe that the $\mathrm{TiO}_{2}$ coating was at least partially crystalline, the dark spots being regions with high scattering efficiency when the Bragg condition was satisfied when the electron micrograph was taken. With higher magnification, the apparently microcrystalline morphology of the $\mathrm{TiO}_{2}$ layer can be seen even more clearly in (c).

To put our understanding on a firm theoretical ground, as well as for a more quantitative comparison with experiment, we have carried out a first-principles calculation for the doubly coated spheres system. The aim of the calculation is the solution of the electrostatic equation for the potential function $\phi(\mathbf{r})$ :

$$
\nabla \cdot[\epsilon(\mathbf{r}) \nabla \phi(\mathbf{r})]=0,
$$

where the dielectric constant

$$
\epsilon(\mathbf{r})=\epsilon_{l}\left[1-\sum_{i}^{3} \eta_{i}(\mathbf{r}) / s_{i}\right],
$$

$s_{i}=\epsilon_{l} /\left(\epsilon_{i}-\epsilon_{l}\right), \epsilon_{i}$ is the dielectric constant of the $i$ th 

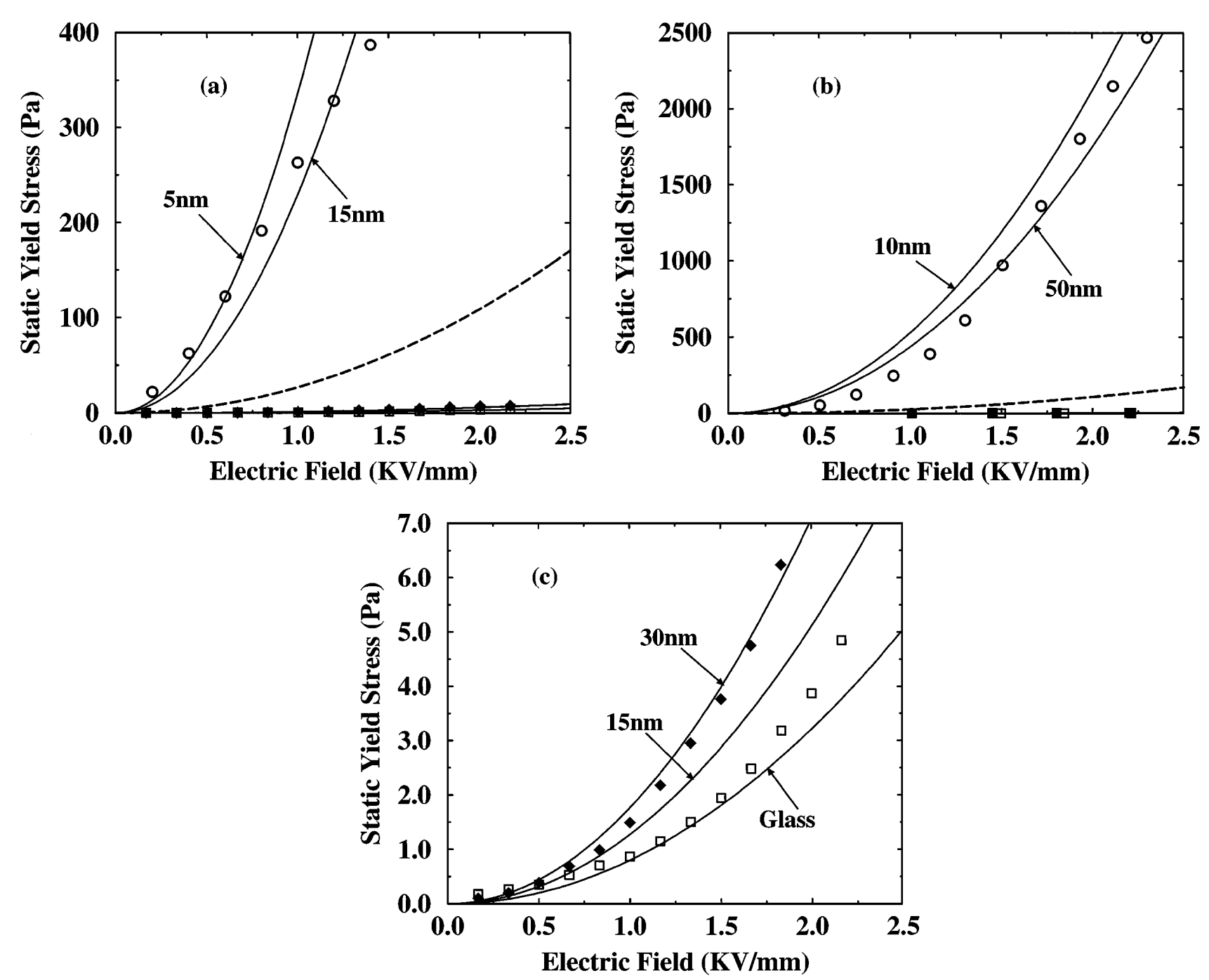

FIG. 2. The measured (symbols) and calculated (lines) static yield stress of ER fluid using (a) $1.5 \mu \mathrm{m}$ doubly coated particles, (b) $50 \mu \mathrm{m}$ doubly coated particles, and (c) expanded scale of (a), uncoated particles (open squares) and singly coated with $\mathrm{TiO}_{2}$ only (solid squares.) As explained in the text, the solid line is yield stress obtained from first-principles calculations using known dielectric constants, and measured geometric information of the particles. Because of the variation of $\mathrm{TiO}_{2}$ coating thickness as seen in Figs. 1(b) and 1(c), the calculation was done for a range of $\mathrm{TiO}_{2}$ thickness, as labeled. As a comparison, we have also calculated for the case of solid $\mathrm{TiO}_{2}$ particles of the same sizes (dashed line) showing that the doubly coated particles have far superior static yield stress.

material component, and $\eta_{i}(\mathbf{r})$ is the characteristic function, taking the value 1 in the region ofmaterial component $i$, and 0 otherwise. The characteristic functions contain all the microstructural information of the system. The boundary condition imposed on Eq. (1) is that $\phi=0$ at $z=0$, and $\phi=L$ at $z=L$, with $L \rightarrow \infty$. By following the formalism developed by Bergman and Milton [7], the solution of Eq. (1) directly yields the effective dielectric constant $\bar{\epsilon}$ of the system:

$$
\bar{\epsilon}=\epsilon_{l}\left(1-\frac{1}{V} \sum_{i} \frac{1}{s_{i}} \int d \mathbf{r} \eta_{i}(\mathbf{r}) \frac{\partial \phi(\mathbf{r})}{\partial z}\right),
$$

where $|\phi\rangle=\left[1-\sum_{i} \Gamma_{i} / s_{i}\right]^{-1}|z\rangle$ is the formal solution to Eq. (1), with

$$
\Gamma_{i}=\int d \mathbf{r}^{\prime} \eta_{i}\left(\mathbf{r}^{\prime}\right) \nabla^{\prime} G\left(\mathbf{r}, \mathbf{r}^{\prime}\right) \cdot \nabla^{\prime},
$$

and $G\left(\mathbf{r}, \mathbf{r}^{\prime}\right)=1 / 4 \pi\left|\mathbf{r}-\mathbf{r}^{\prime}\right|$ being Green's function for the Laplacian operator, and $V$ denotes the sample volume. Note that $\bar{\epsilon}$ is only a function of materials and microstructures.

The effective dielectric constant is the key to the determination of the ground state structure of the system in the high-field limit, as well as the mechanical ER properties. That is, since the free energy density of the system in the high-field regime (i.e., where the electrostatic energy of each particle $\gg$ the thermal energy) is given by $f=-\bar{\epsilon} E^{2} / 8 \pi$, the ground state structure is determined by maximizing $\bar{\epsilon}$ with respect to the coordinates of the solid particles. For doubly coated spheres, the body- 
centered tetragonal (bct) structure is the optimal structure, same as that for the bare sphere case [8-12]. To calculate the static yield stress, one just needs to perturb the system away from the ground state by applying a shear deformation to the system that is perpendicular to the applied field direction. Under shear, the $c$ axis of the bct structure is both tilted by an angle $\theta$ with respect to the field as well as elongated [9]. The stress-strain relation is directly given by $\partial f / \partial \theta$ versus $\theta$. The static yield stress is then defined as the maximum stress value beyond which the stress decreases with increasing strain, i.e., the solid structure becomes unstable. Given the geometric information on the particles (diameter and coating thickness) and the known dielectric constants of the materials involved (the values of $\epsilon$ for $\mathrm{TiO}_{2}$ and silicon oil used are, respectively, 85 and 2.5) we can obtain from this calculation the predicted yield stress, at various $\mathrm{TiO}_{2}$ thickness, plotted as solid lines in Figs. 2(a) and 2(b). [The dielectric constant for $\mathrm{TiO}_{2}$, depending on its crystalline orientation with respect to the applied electric field, is 85 or 170 . Here, for lack of information supporting a highly crystalline state, we use the lower value.] We see good agreements (to the extent we can expect from such complex systems) for both sizes. Particles with more uniform coating thickness and monodispersed in size will likely lead to even better agreement between theory and experiment. Even though technically the calculation should apply only in the high-field regime, we found that the theoretical quadratic field dependence can adequately describe the experimental finding even at low fields. Using the same theoretical model, we have also calculated the yield stress for bare glass particles and $\mathrm{TiO}_{2}$ coated particles (without inner metallic coating). The results, together with our experimental results for such particles, are shown in Fig. 2(c), for the $1.5 \mu \mathrm{m}$ particles. As a reference, we also calculated (but no experimental result) the yield stress expected for $\mathrm{TiO}_{2}$ particles of the same size [shown as dashed lines in Figs. 2(a) and 2(b)]. The efficacy of the doubly coated DMP system is clear.

A simple physical picture emerges from our results above. ER yield stress is closely related to the electrostatic energy of the system. For a given applied electric field, the electrostatic energy can be maximized by field distribution and dielectric constant of the materials. The metallic coating of the DMP's limits the field distribution to the dielectric areas occupied by $\mathrm{TiO}_{2}$ and silicon oil. The thin $\mathrm{TiO}_{2}$ coatings ensure small separations, thus producing high internal fields. The dramatic ER enhancement is the result.

In conclusion, the propose and experimentally demonstrated a new type of ER system using doubly coated particles. We present a simple physical picture that provides intuitive understanding using electrostatic energy arguments. This is further confirmed by a first-principles calculation that can account for quantitatively all of our experimental findings. We believe this coupling of fabrication, characterization, and physical understanding on a firm theoretical basis will lead to further improvement towards the ultimate goal of practical applications.

We thank Chi Ming Chan and David Barber for helpful discussions in electroless wet plating and sol-gel techniques. We also thank Supapan Seraphin, Ning Wang, and Kwok Kwong Fung for obtaining the cross sectional electron micrographs, as well as for helpful discussions. We acknowledge Ji Zhou and Keding Xia for technical support and Yee Wai Leung for assistance in conducting the experiment. This work is supported by HKUST Research Infrastructure Grant No. RI 93/94.SC09, and the William Mong Solid State Cluster Laboratory.

*Permanent address: Department of Physics, Jiaotung University, Shanghai, People's Republic of China.

[1] K. O. Havelka and F. E. Filisko, Progress in Electrorheology (Plenum Press, New York, 1995).

[2] K.J. Korane, Mach. Des. 63, 52 (1991); G. Goldstein, Mech. Eng. 112, 48 (1990).

[3] ER fluid with insulating metallic particles has been reported by A. Inoue, in Electrorheological Fluids, edited by J.D. Carlson, A.F. Sprecher, and H. Conrad (Technomic Publishing Co., Lancaster, 1989), p. 176. Experimentally, the shear stress was found to be $272 \mathrm{~Pa}$ at $2 \mathrm{kV} / \mathrm{mm}$. The added choice of a core material, plus the quantitative theoretical and experimental studies, distinguishes our work from that of Inoue's. This leads to a deeper, quantitative understanding of the effect and opens a path of continuous improvement. As a result our present experimental result [Fig. 2(a)] already shows a $7 \times$ improvement (at $2 \mathrm{kV} / \mathrm{mm}$ ) compared to that of Inoue's.

[4] A calculation for a chain of metallic particles separated by finite spacings has been reported by R. A. Anderson, Langmuir 10, 2917 (1994).

[5] J. W. Severin, R. Hokke, H. Vanderwel, and G. deWith, J. Electrochem. Soc. 140, 682-687 (1983).

[6] C. J. Briker and G.W. Scherer, Sol-Gel Science: The Physics and Chemistry of Sol-Gel Processing (Academic Press, New York, 1990).

[7] D. J. Bergman, in Solid State Physics, edited by H. Ehrenreich and D. Turnbull (Academic Press, New York, 1992), Vol. 46, p. 147; G. W. Milton, Appl. Phys. A 26, 1207 (1981); G. W. Milton, J. Appl. Phys. 52, 5286 (1980).

[8] R. Tao and J. M. Sun, Phys. Rev. Lett. 67, 398 (1991); R. Tao and J. M. Sun, Phys. Rev. A 44, R6181 (1991).

[9] H. Ma, W. Wen, W. Y. Tam, and P. Sheng, Phys. Rev. Lett. 77, 2499 (1996).

[10] G. Bossis, H. Clercx, Y. Grasselli, and E. Lemaice, in Electrorheological Fluids, edited by R. Tao and G. D. Roy (World Scientific, Singapore, 1994), p. 153; H. Clercx and G. Bossis, Phys. Rev. E 48, 2721 (1993).

[11] R. Friedberg and Y. K. Yu, Phys. Rev. B 46, 6582 (1992).

[12] L. C. Davis, Phys. Rev. A 46, R719 (1992). 